Submitted to Ap.J.

\title{
A Weak Gravitational Lensing Analysis of Abell 2390
}

\author{
G. Squires ${ }^{1}$ N. Kaiser ${ }^{2,5}$, G. Fahlman ${ }^{3,5}$, A. Babul ${ }^{4}$ and D. Woods ${ }^{3}$
}

\begin{abstract}
We report on the detection of dark matter in the cluster Abell 2390 using the weak gravitational distortion of background galaxies. We find that the cluster light and total mass distributions are quite similar over an angular scale of $\simeq 7^{\prime}\left(1 h^{-1} \mathrm{Mpc}\right)$. The cluster galaxy and mass distributions are centered on the cluster $\mathrm{cD}$ galaxy and exhibit elliptical isocontours in the central $\simeq 2^{\prime}\left(280 h^{-1} \mathrm{kpc}\right)$. The major axis of the ellipticity is aligned with the direction defined by the cluster $\mathrm{cD}$ and a "straight arc" located $\simeq 38$ " to the northwest. We determined the radial mass-to-light profile for this cluster and found a constant value of $(320 \pm 90) h M_{\odot} / L_{\odot V}$, which is consistent with other published determinations. We also compared our weak lensing azimuthally averaged radial mass profile with a spherical mass model proposed by the CNOC group on the basis of their detailed dynamical study of the cluster. We find good agreement between the two profiles, although there are weak indications that the CNOC density profile may be falling more steeply for $\theta \geq 3^{\prime}\left(420 h^{-1} \mathrm{kpc}\right)$.
\end{abstract}

\footnotetext{
${ }^{1}$ Center for Particle Astrophysics, University of California, Berkeley, CA 94720 USA

${ }^{2}$ Canadian Institute for Advanced Research and Canadian Institute for Theoretical Astrophysics, University of Toronto, 60 St. George St., Toronto, Ontario, Canada M5S 1A7

${ }^{3}$ Department of Geophysics and Astronomy, University of British Columbia, 2219, Main Mall, Vancouver, BC, Canada V6T 1Z4

${ }^{4}$ Department of Physics, New York University, 4 Washington Place, Room 525, New York, New York, USA $1003-6621$

${ }^{5}$ Visiting Astronomer, Canada-France Hawai'i Telescope. Operated by the: National Research Council of Canada, le Centre National de la Recherche Scientifique de France and the University of Hawai'i
} 
Subject headings: cosmology: observations - dark matter - gravitational lensing - galaxy clusters - large scale structure of universe

\section{Introduction}

Understanding the nature of the mass distribution in clusters of galaxies is a key cosmological issue. Typically, three independent methods to probe the cluster mass distribution have been applied: 1) applications of the virial theorem to the cluster galaxy velocity distribution, 2) analyses of the X-ray emission of the diffuse, intracluster gas and 3) mass determinations based on the gravitational lens distortion of background objects by the foreground cluster. The latter technique, the analyses of both the strong as well as the weak gravitationally induced distortions in the images of faint background galaxies, is particularly attractive as it does not require any assumptions about the geometry or the dynamical of the clusters, with the weak distortions being especially suited for mapping the cluster mass distribution out to large radii.

Over the past few years, there has been considerable activity to develop a theoretical framework for determining the mass distribution in the cluster based on a measured set of gravitationally distorted galaxy shapes (eg. Tyson et al. 1990; Kaiser \& Squires 1994; Schneider 1994; Kaiser et al. 1995; Schneider \& Seitz 1995; Seitz, C., \& Schneider 1995; Seitz, S., \& Schneider 1995, Squires \& Kaiser 1995). Several groups have developed algorithms and software designed to measure and analyze very small distortions expected in the cluster outskirts (Kaiser, Squires \& Broadhurst 1995; Bonnet \& Mellier 1995; Fischer \& Tyson 1996). These techniques have been applied to the central $\simeq 1 h^{-1} \mathrm{Mpc}$ of several galaxy clusters (eg. Tyson et al. 1990; Fahlman et al. 1994; Bonnet et al. 1994; Tyson \& Fischer 1995; Squires et al. 1995).

For a few clusters, the results of the weak lensing analyses has compared with the results of X-ray analyses as well as detailed spectroscopic studies. There is no apriori reason to expect an agreement between the different analyses and in fact, such joint analyses can potentially enable to us learn much more about the relative distributions and the dynamical states of the gas, galaxies and dark matter in the clusters than is currently known. Indeed, in the central regions of some clusters (eg. A2218 and A1689), the mass inferred from the presence of (strong) lensing features exceeds that derived from the X-ray data under the standard assumption of pressure supported hydrostatic equilibrium by a factor of $\simeq 2-3$ (Miralda-Escudé \& Babul 1995). A similar discrepancy 
also arises in galaxy cluster MS 1224, where the weak lensing inferred mass is $\simeq 2$ times larger than the mass determined from a virial analysis of the cluster galaxies Fahlman $e$ al. 1994; Carlberg et al. 1994). Also, in a joint lensing/X-ray analysis of A2218 (Squires et al. 1995), the lensing mass profile was found to be systematically a factor of $\simeq 2$ larger than the X-ray mass profile, although the two are formally in agreement within the $95 \%$ confidence level. A recent analysis based on ASCA data confirmed this discrepancy (Loewenstein 1996). Conversely, a similar analysis of the cluster A2163 yielded good agreement between the weak lensing and X-ray mass estimates (Squires et al. 1996).

In this paper, we discuss the dark matter and galaxy distributions in the cluster of galaxies Abell 2390. Abell 2390 is an Abell richness class 1 cluster (Abell et al. 1989) at redshift $z=0.2279$ with a measured line of sight velocity dispersion $\sigma=1093 \mathrm{~km} / \mathrm{s}$ (Carlberg et al. 1995). Abell 2390 has an X-ray luminosity of $L_{x}=9.77 \times 10^{44} \mathrm{ergs} / \mathrm{s}$ $(\mathrm{H}=75 \mathrm{~km} / \mathrm{s} / \mathrm{Mpc}$ ) in the $2-6 \mathrm{keV}$ energy range (Kowalski et al. 1984) and $L_{x}=4.67 \times 10^{44} \mathrm{ergs} / \mathrm{s}$ in the $0.7-3.5 \mathrm{keV}$ range (Ulmer et al. 1986). A2390 has been the focus of several studies (eg. Pello et al. 1991, Kassiola et al. 1992, Narasimha \& Chitre 1993) seeking to account for the observed lensing features such as the "straight arc" located approximately 38" away from the central galaxy (एPello et al. 1991).

In this analysis, the total mass distribution in A2390 is determined using the weak gravitational distortion of faint background images induced by the cluster. The data reduction and analysis procedure used here is very similar to that we employed in our analysis of A2218 (Squires et al. 1995) and A2163 (Squires et al. 1996) and we refer to those papers for further detail. Here we will concentrate mainly on the results. We also compare our results for the morphology of the mass distribution and the estimate for the total mass with published results from galaxy spectroscopy and X-ray analyses.

\section{Data Acquisition and Analysis}

The cluster was observed at the 3.6m Canada-France-Hawai'i telescope on the nights of 1994 June 6-9. The detector used was the 2048 x 2048 Loral 3 CCD at prime focus with a pixel size of 0 '.207. For the cluster Abell 2390, we obtained $5 \times 15$ minute exposures in the I-band and $2 \times 30$ minute exposures in $\mathrm{V}$ with seeing conditions of $\mathrm{FWHM}=0^{\prime \prime} .6$ and $0^{\prime \prime} .8$ in I and $\mathrm{V}$ respectively. We observed the central $7^{\prime}$ of the cluster, covering a square of side $\simeq 1 h^{-1} \mathrm{Mpc}$ at the cluster redshift. 
A detailed discussion of the observations and data reduction are described in Squires et al. 1995. Very briefly, each bias subtracted image was divided by a median twilight flat. The residual rms scatter in the sky background on the individual images was 23.9 magnitudes per square arcsecond in I, and 25.3 magnitudes per square arcsecond in V. The data was calibrated against photometric standards in the globular clusters M92 and NGC 4147 (unpublished photometry from Davis 1990; see also Stetson and Harris 1988 and Odewahn et al. 1992) and Landolt (1992) standards in SA110. Color terms were found to be unnecessary in the transformation and the $\mathrm{I}$ and $\mathrm{V}$ zero points were determined with a formal error of less than 0.005 mag.

We detected objects with a significance of $4 \sigma$ over the local sky background in each frame and selected objects that were detected on least two of the images in each waveband independently. We formed master catalogues of 1828 objects in I and 1255 in $\mathrm{V}$ which are complete to $\simeq 23.5$ and $\simeq 24.0$ in $\mathrm{I}$ and $\mathrm{V}$ respectively. We measured the position, shape and brightness parameters for all of the objects using our standard procedure (Kaiser, Squires \& Broadhurst 1995).

\section{Cluster Light Distribution}

Combining the V-and I-band observations, we determined the color of objects detected in both wavebands. We identified a red sequence of objects with mean color of $V-I \simeq 1.7$ (uncorrected for reddening) at the bright end which we classified as cluster galaxies. To extract this color selected cluster galaxy sample, we fitted a linear model to the color sequence and selected bright objects $(I<21)$ with color within 0.2 magnitudes of the mean.

To obtain a qualitative description of the cluster light distribution, we calculated the galaxy light and galaxy surface number density. We employed the color selected sample and display as contour plots and 3D surface plots the cluster galaxy light distribution and surface number density in Figure 2. The distributions have been smoothed with a Gaussian filter with scale 0.5 (this scale was chosen match the smoothing used in the lensing analysis). The galaxy number and light distributions are very similar. The dominant structure in both distributions is centered on the giant elliptical galaxy and exhibits some ellipticity in the central regions, with the major axis oriented in the direction towards the the straight arc located $\simeq 38^{\prime \prime}$ to the northwest of the central galaxy. There is a small secondary peak approximately $4^{\prime}$ to the northwest of the $\mathrm{cD}$ 
galaxy that appears in both the number and luminosity weighted plots. The height of the secondary peak is $\simeq 20 \%$ of the amplitude of primary concentration in the smoothed light map.

We estimated the total light content in the cluster in two ways. First, we formed a lower bound on the cluster light by only including the light associated with galaxies having the mean cluster galaxy color. This excludes cluster members with color outside the main cluster color sequence as well as galaxies with magnitudes beyond the faint-end limit. Secondly, we calculated the light in the cluster by adding the light associated with all the galaxies detected in the image. Since this sample includes both cluster as well as field galaxies, the resulting measure of the light provides an upper limit to the light content of the cluster, down to the limiting magnitude of the catalogue. We calculated the zero redshift $\mathrm{V}$-band luminosity by $L_{V}=10^{0.4\left(M_{V \odot}-V+D M+K+A_{V}\right)} L_{\odot V}$ where $M_{V \odot}=4.83$ is the total solar $\mathrm{V}$ magnitude. We applied a K-correction of $K=0.5$ as suggested by Coleman, Wu and Weedman (1980). Since A2390 is at redshift $z=0.2279$, its distance modulus is $D M=39.28$ (for $\Omega=1$ and $h=1$ ) and we used an extinction correction of $A_{V}=0.25$.

The cumulated light profiles are shown in Figure 1. The solid line shows the results using all the galaxies in the images, while the dashed line shows the light profile computed from the color selected sample. The slope and amplitude of the light of the full galaxy sample exceeds that of the cluster selected subsample and for $\theta \geq 100^{\prime \prime}$, the

former is a factor of $\geq 1.5$ greater. The true cluster light profile is likely to lie in between the two estimates. Regardless, in computing quantities such as the cluster mass-to-light ratio, we adopt a conservative approach and use the higher of the two profiles.

\section{Lensing Analysis}

We identified galaxies as objects having half-light radius greater than 1.2 times the mean stellar half-light radius and applied a magnitude cut of $21<I<24$. The bright magnitude limit was chosen, somewhat arbitrarily, to exclude cluster and foreground galaxies, but the results are very insensitive to the actual threshold level. With these cuts, we obtained 922 galaxies for this analysis. We present here the I-band data for the lensing analysis as the seeing conditions were significantly better than the V-band observations (although the V-band data was used as a consistency check). To convert the measured galaxy shapes into estimates of the cluster surface density, we corrected for 

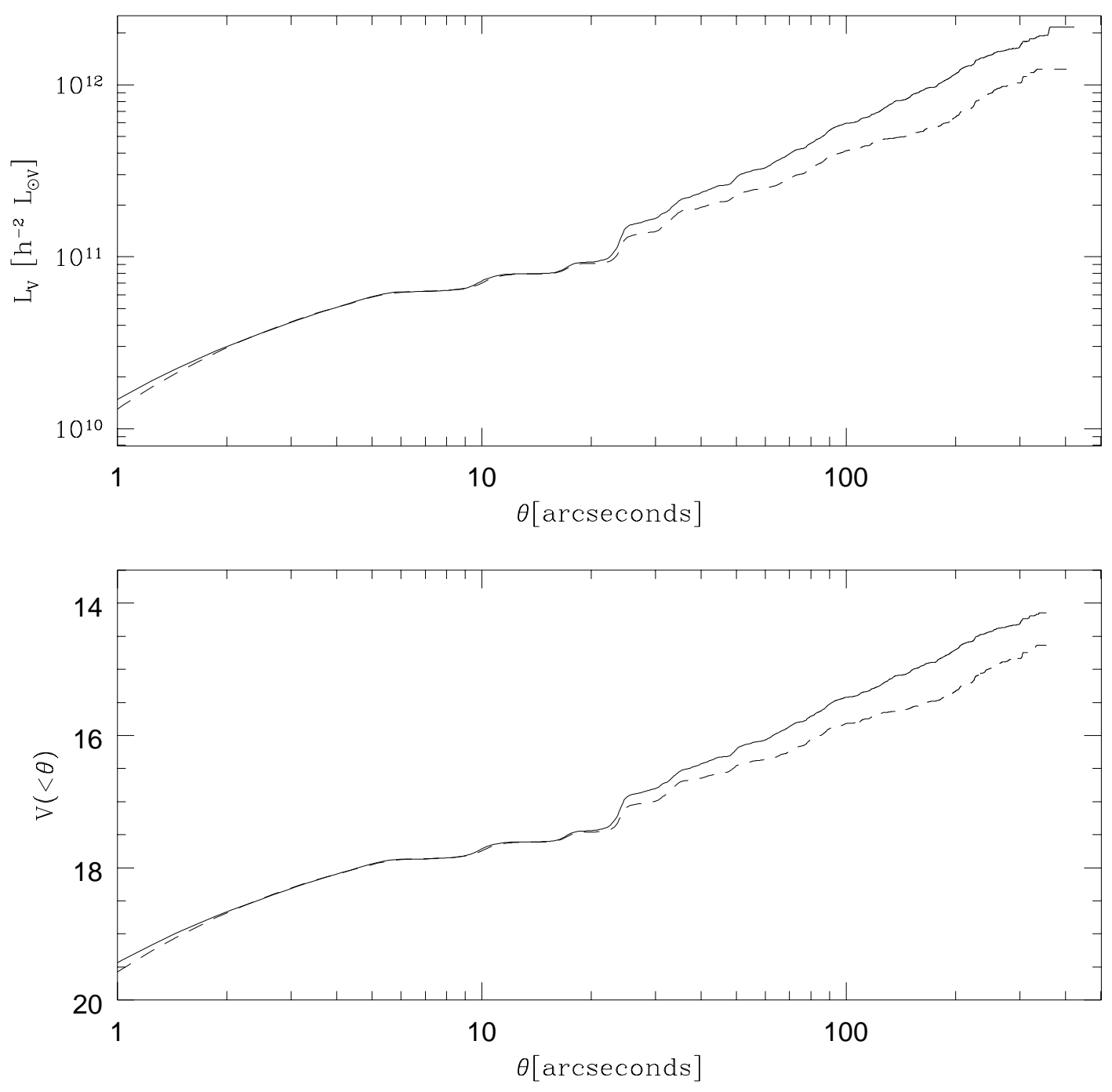

Fig. 1.- The cumulated V-magnitude and $L_{V}$ luminosities from the central elliptical galaxy. The solid lines come from adding the light from all galaxy candidate objects in the field. The dashed line is for the color selected sequence. 
the effects of anisotropy in the point spread function (psf) due to wind-shake, guiding errors, etc., and the smoothing due to seeing using the procedure described in Kaiser, Squires \& Broadhurst (1995).

We used the seeing and psf-corrected galaxy shape measurements to determine the surface mass density in the cluster and display the results in Figure 2. The surface density reconstruction was determined using the maximum probability extension to the original Kaiser \& Squires (1993) algorithm (Squires \& Kaiser 1995). We employed 15 wavemodes and a regularization parameter of $\alpha=0.05$ although we note that the results are quite insensitive to variations in $\alpha$ by a factors of $\simeq 5$.

The peak of the mass concentration coincides with the central giant elliptical galaxy and is significant at the $\simeq 6 \sigma$ level. For $\theta \geq 1^{\prime}$, the mass isocontours are elongated in the southeast-northwest direction, in alignment with the direction defined by the central galaxy and the straight arc. There is an extension towards the north at a distance of $\simeq 2^{\prime}$ of the central galaxy which lies in the direction of the overdensity that appeared in the cluster number and light distributions. The peak itself (if it exists in the mass distribution) is not resolved. This is not unreasonable given the number of galaxies used in this reconstruction: assuming that mass scales directly with light, then we would only expect to detect the secondary mass fluctuation at the $\simeq 1 \sigma$ level. Thus, with the surface density of galaxies available here, we can not map with high statistical significance the mass associated with small fluctuations in the cluster galaxy distribution.

To determine the total mass in the cluster, we employed the statistic (Kaiser et al. 1995)

$$
\zeta\left(\theta_{1}, \theta_{2}\right)=2\left(1-\theta_{1}^{2} / \theta_{2}^{2}\right)^{-1} \int_{\theta_{1}}^{\theta_{2}} d \ln (\theta)\left\langle\gamma_{t}\right\rangle
$$

which measures the mean dimensionless surface density interior to $\theta_{1}$, relative to the mean in an annulus $\theta_{1}<\theta<\theta_{2}$. To convert $\zeta$ into a physical quantity, we estimated $\Sigma_{\text {crit }}=\left(4 \pi G D_{l} \beta\right)^{-1}$ where $D_{l}$ is the angular diameter distance to the lens and $\beta=\max \left(0,\left\langle 1-w_{l} / w_{s}\right\rangle\right)$. In an Einstein de-Sitter universe with $\Omega=1$, the comoving distance $w$ is defined as $w=1-1 / \sqrt{1+z}$. We estimated $\beta$ by an extrapolation of the faint redshift surveys (Lilly 1993; Tresse et al. 1993) to fainter magnitudes and find, for $\mathrm{A} 2390, \Sigma_{\text {crit }}=(6.5 \pm 0.6) \times 10^{15} h M_{\odot} / \mathrm{Mpc}^{2}$.

Using the estimate of $\Sigma_{\text {crit }}$, we convert the $\zeta$ estimates into a mass. In Figure 3 we display the radial mass profile of Abell 2390 and the radial mass-to-light ratio. We 


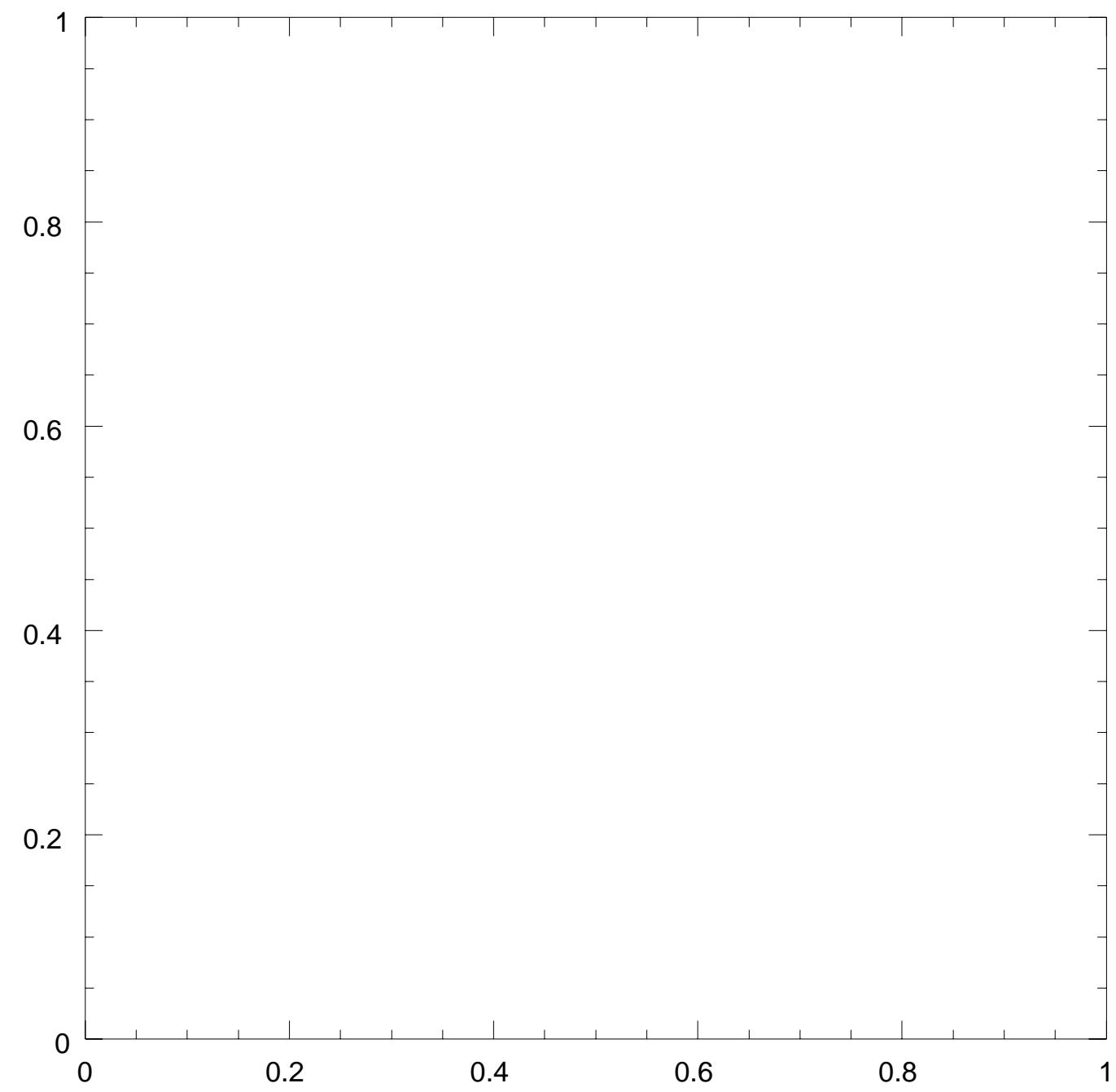

Fig. 2.- The bottom two rows show the luminosity and number weighted distributions of the color selected cluster galaxy sample. The top row is the reconstructed mass distribution. The distributions have been smoothed with a Gaussian filter with scale 0.5. In the contour plots, North is to the right; East is up. The box size is $7^{\prime}=966 h^{-1} \mathrm{kpc}$. This figure can be obtained at ftp://magicbean.berkeley.edu/pub/squires/a2390/massandlight.ps.gz 
also plot the mass interior to the position of the straight arc in a bi-modal mass model proposed by Pierre et al. (1995) as well as display the predictions from two simple models for the cluster mass profile. The solid line shows the prediction for the mass profile from a singular isothermal sphere with the observed velocity dispersion (Carlberg et al. 1995), correcting for the mass contained in the control annulus at each radial bin. This mass profile does not appear to agree with the weak lensing mass profile, predicting little mass for $\theta \geq 150^{\prime \prime}$, although uncertainties in the weak lensing estimates are sufficiently large that the singular model cannot be excluded with a high statistical significance. The dashed line corresponds to a model proposed on the basis of the CNOC analysis of the cluster galaxy velocity distribution (Carlberg et al. 1996) under the assumption that the light traces the mass: $\rho(r)=\rho_{0} a / r(r+a)^{3}$. We have projected the three-dimensional density model and corrected for the mass in the control annulus. The lensing results and the CNOC model are consistent within the $95 \% \mathrm{CL}$ at all radii probed here. It is worth noting, however, that weak lensing profile is rising systematically more steeply than the CNOC profile for $\theta \geq 150^{\prime \prime}$.

We caution that in the very central regions, the lensing results should be interpreted with care. First, the analysis performed here is strictly only valid in the weak lensing regime; the presence of the straight arc at a radius of $\simeq 38^{\prime \prime}$ makes this assumption dubious in the innermost arcminute or so. Secondly, detailed analyses of other clusters seem to indicate that the lensing signal in the central regions $\left(\theta \leq 1^{\prime}\right)$ is most susceptible to dilution due to the contamination of our background galaxy catalogue by foreground and cluster galaxies. Since the results presented here were determined using only 922 galaxies, any contamination would significantly degrade the lensing signal. Fortunately, the dilution effect does not appear to significant beyond a radius of $\simeq 1^{\prime}$. One way to address this is to select only galaxies redder than the cluster color sequence - these should mainly lie behind the cluster. We repeated the above analysis using such a sample and found consistent results, although the associated uncertainties are substantially larger since the number of galaxies in the sample is much smaller.

Using the cluster light and the lensing mass profiles, we computed the cluster mass-to-light ratio as a function of radius. To account for the subtraction of the material in the control annulus in the mass estimator, we formed an analogous quantity, $\zeta_{L}$, for the light which is the mean light surface density interior to a given radius, relative to the mean in the control region. Under the assumption that mass traces the light the cluster light and the lensing mass profiles are consistent with this assumption, the ratio of the $\zeta$ values based on the observed shear and $\zeta_{L}$ forms an unbiased estimate 
of the mass-to-light ratio in the cluster. The light estimate comes from using all the light associated with the galaxies detected in the image (which, as we have discussed previously, includes contributions from galaxies not associated with the cluster). We find a mass-to-light ratio of $M / L_{V}=(320 \pm 100) h M_{\odot} / L_{V \odot}$ at $\theta \approx 2.5 \approx 0.345 h^{-1} \mathrm{Mpc}$, with the radial profile being consistent with a constant $M / L_{V}$ across the cluster.

\section{Discussion}

We have mapped the light and mass distributions in the cluster of galaxies A2390 over a scale of $\simeq 250^{\prime \prime}$ from the cluster center. The projected light distribution was determined using a color selected galaxy catalogue. The total radial light profile was calculated using all of galaxies detected, which we expect should include some contributions from galaxies not associated with the cluster. To map the mass distribution,
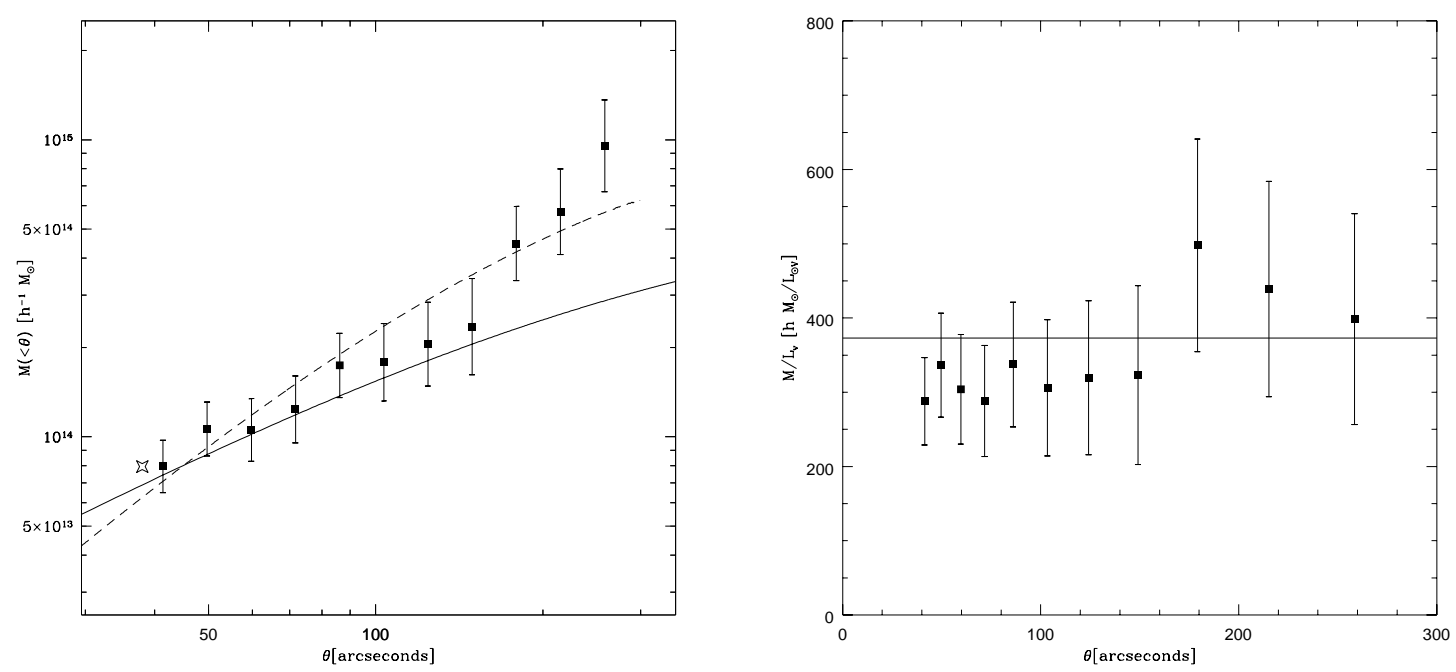

Fig. 3.- Left panel: the radial mass profile. The solid line is the prediction for an isothermal model with the measured velocity dispersion of $\sigma=1093 \mathrm{~km} / \mathrm{s}$ and the dashed line is the CNOC model (Carlberg et al. 1996). Both predictions have been corrected for matter in the control annulus to facilitate direct comparison with the lensing data. The " $\mathrm{x}$ " is the mass estimated by Pierre et al. (1995) using a bimodal model. Right panel: the radial mass-to-light profile, determined by taking the ratio of the mean surface mass and light densities as a function of radius, relative to the mean in the control annulus. 
we measured the shapes of faint galaxies which have been gravitationally distorted, correcting for systematic effects in the shape determinations such as psf anisotropy and seeing.

The projected light and mass distributions are displayed in Figure 2. Both the light and mass distributions are centered on the $\mathrm{cD}$ galaxy. The light and mass isocontours are elliptical in the central region, with the major axis running from the southeast to northwest. There is a smaller, secondary concentration in both the cluster galaxy and light distributions located approximately $4^{\prime}$ to the northwest of the cluster center. The mass map gives the impression that the mass distribution is extended in the direction of the secondary concentration seen in the cluster light distribution but there is no corresponding peak in the mass map. As we noted previously, this is not surprising given the number of galaxies used in constructing the mass map.

Several authors (eg. Pello et al. 1991; Kassiola et al. 1992) have attempted to account for the lensing features seen in A2390 by suggesting that the cluster has bi-modal mass distribution, with a small secondary mass concentration located near the observed arc position. This model is further supported by the cluster X-ray emission, which shows elliptical isocontours centered on the cluster cD galaxy as well as a significant secondary peak located $40^{\prime \prime}$ to the northwest of the cluster center (Pierre et al. 1995). The weak lensing mass map presented here is not inconsistent with the notion of a bi-modal mass distribution. As we have noted previously, the small-scale results from the weak lensing analysis are subject to greater uncertainties arising from the combined effects of nonlinearity in the lensing, contamination by cluster galaxies, and the statistical uncertainties due to the small number of galaxies used in the mass reconstructions.

We calculated the mass-to-light ratio as a function of radius (Figure 3) and found a constant value of $M / L_{V}=(320 \pm 90) h M_{\odot} / L_{\odot V}$, although we have noted that the mass estimates may be biased downwards in the innermost $\simeq 1^{\prime}$ due to contamination by faint cluster galaxies. In the course of their study of several intermediate redshift cluster, the CNOC group derived a mass-to-light ratio, K-corrected to redshift zero, of $M / L_{r}=(331 \pm 54) h M_{\odot} / L_{\odot r}$ for the cluster. We convert the Gunn r luminosity into $\mathrm{V}$-band luminosity by applying the transformation $\mathrm{V}-\mathrm{r}=0.16$ (which is typical for a nearby early type galaxy) and find that the CNOC result corresponds to $M / L_{V}=(370 \pm 60) h M_{\odot} / L_{\odot V}$, which is in excellent agreement with out determination.

We furthermore compared the radial mass profile determined from this analysis with the CNOC model. We projected the CNOC 3D mass model and calculated the 
$2 \mathrm{D}$ radial mass profile, correcting for mass in the control annulus to facilitate direct comparison with the lensing results (Figure 31). All of the mass estimates based on the lensing analysis agree within the 95\% CL with the CNOC model (although we note that the CNOC model assumes spherical symmetry and there is some indications that this might be invalid on the scales probed here). At the very largest radius, there is a slight indication that the lensing inferred mass might be falling slower than the CNOC model, although the values are consistent within $2 \sigma$. Larger scale observation of this cluster will prove useful to determine if this trend continues at large radii.

As with many of the weak lensing studies performed to date, this analysis was confined to a relatively small field of view. This has the immediate consequence that, since the mass determinations made by this technique are differential, the masses quoted form a lower bound on the total mass at any radius. The future prospects for this method are exciting as the mass distributions are mapped out to large distances in the cluster halos. Indeed, the technology now exists for such large field lensing observations with the MOCAM $14^{\prime}$ and the $\mathrm{UH} \simeq 0.5^{\circ}$ cameras at CFHT. With the types of observations possible with these instruments, the weak lensing technique offers a unique opportunity to probe many of the outstanding puzzles regarding the dark matter content and distribution in the universe. 


\section{REFERENCES}

Abell, G.O., Corwin, H.G., \& Olowin, R.P. 1989, ApJS, 70, 1

Bonnet, H., Fort, B., Kneib, J.-P., Mellier, Y., \& Soucail, G. 1994, A\&A, 280, L5

Bonnet, H., \& Mellier, Y. 1995, A\&A, accepted

Carlberg, R.G., Yee, H.K.C., \& Ellingson, E. 1995, ApJ, 437, 63

Carlberg, R.G., Yee, H.K.C., \& Ellingson, E. 1995, ApJ, submitted

Carlberg, R.G., Yee, H.K.C., Ellingson, E., Morris, S., Abraham, R., Gravel, P., Pritchet, C., \& Smecker-Hane, T. 1996, in preparation

Davis, L. 1990, private communication

Fahlman, G., Kaiser, N., Squires, G., \& Woods, D. 1994, ApJ, 437, 56

Fischer, P., \& Tyson, J. 1996, in preparation.

Kaiser, N., \& Squires, G. 1993, ApJ, 404, 441

Kaiser, N., Squires, G., \& Broadhurst, T. 1995, ApJ, 449, 460

Kaiser, N., Squires, G., Fahlman, G., \& Woods, D. 1995, in eds.: F. Durret, A. Mazure, J. T.T. Van, Clusters of Galaxies, Editions Frontiières, Gif-sur Yvette Cedex, in press

Kassiola, A., Kovner, I., \& Blandford, R. D. 1992, ApJ, 396, 10

Kowalski, M.P., Cruddace, R.G., Wood, K.S., \& Ulmer, M.P. 1984, ApJS, 56, 403

Landolt, A. U. 1992, A\&A, 1992, 104, 340

Lilly, S.J. 1993, ApJ, 411, 501

Loeb, A., \& Mao, S. 1994, ApJ, 435, 109

Loewenstein, M. 1996, in proceedings of the 2nd international conference sponsored by UCLA on Sources and Detection of Dark Matter in the Universe, in press

Miralda-Escudé, J. \& Babul, A. 1995, ApJ, 449, 18

Narasimha, D., \& Chitre, S.M. 1993, Ap \& A, 14, 121 
Odewahn, S.C., Bryja, C., \& Humphreys, R.M. 1992, PASP, 104, 553

Pello, R., Sanahuja, B., Le Borgne, J., Soucail, G., \& Mellier, Y. 1991, ApJ, 366, 405

Pierre, M., Le Borgne, J.F., Soucail, G., \& Kneib, J.P. 1995, preprint

Schneider, P. 1994, preprint

Schneider, P., \& Seitz, C. 1995, A\&A, 294, 411

Seitz, C., \& Schneider, P. 1995, A\&A, 297, 287

Seitz, S., \& Schneider, S. 1995, preprint

Squires, G., \& Kaiser, N. 1995, ApJ, submitted

Squires, G., Kaiser, N., Babul, A., Fahlman, G., Woods, N., Neumann, D. M., \& Böhringer, H. 1995, ApJ, accepted

Squires, G., Neumann, D.M., Kaiser, N., Arnaud, M., Babul, A., Böhringer, H., Fahlman, G., \& Woods, D. 1996, in preparation

Stetson, P. B. \& Harris, W.E. 1988, A\&A, 96, 909

Tresse, L., Hammer, F., le Fevre, O., \& Proust, D. 1993, A\&A, 277, 53

Tyson, J., Valdes, F., \& Wenk, R. 1990, ApJ, 349, L19

Tyson, J., \& Fischer, P. 1995, ApJ, 446, 55

Ulmer, M.P., Kowalski, M.P., \& Cruddace, R.G. 1986, ApJ, 303, 162

This preprint was prepared with the AAS LATEX macros v4.0. 